\title{
INFLUENCIA DEL USO EXCESIVO DE LAS REDES SOCIALES EN EL RENDIMIENTO ACADÉMICO DE LOS ESTUDIANTES DEL III CICLO DE LA FACULTAD DE MEDICINA HUMANA DE LA UNIVERSIDAD NACIONAL SAN LUIS GONZAGA DE ICA, 2017- I
}

Influence of excessive use of the social networks in the academic performance of students of the III cycle of the Faculty of Human Medicine of the San Luis Gonzaga Ica National University, 2017- I

Juana Maribel Arroyo Hernández ${ }^{1}$ a,b

1 Hospital Santa María del Socorro, Ica, Perú

a Médico Cirujano b. Magister en Salud Pública.

\section{RESUMEN}

La participación en redes sociales es cada vez más frecuente en los jóvenes de nuestra comunidad. Se considera que este uso puede repercutir en el rendimiento académico precisamente en quienes desempeñan labores académicas y particularmente en estudiantes universitarios. Objetivo: Determinar si el uso de redes sociales influye en el rendimiento académico de los estudiantes del III Ciclo de la Facultad de Medicina Humana de la Universidad de la Universidad Nacional San Luis Gonzaga de Ica, 2017-I. Material y Métodos: Se contó con la participación de setenta estudiantes quienes voluntariamente aceptaron el llenado de un cuestionario que recabo información sobre tres aspectos: Uso de redes sociales, aspectos socio demográficos y adicción a las redes sociales. Además, se contó con la base de datos sobre calificaciones obtenidas por los participantes en un ciclo académico, y se correlacionaron estadísticamente las variables de estudio. Resultados: La totalidad de participantes manifestó participar en redes sociales (100\%), de las redes consultadas, la denominada Facebook es la más empleada (70\%). Se halló relación inversa entre el uso excesivo de redes sociales y el desempeño académico de los participantes $(p=0,000)$. Conclusiones: Existe relación inversamente proporcional entre el uso de redes sociales y el rendimiento académico en estudiantes del III ciclo de la Facultad de Medicina Humana de la Universidad Nacional San Luis Gonzaga de Ica. 2017-I, a mayor uso de redes sociales, el rendimiento académico es menor.

Palabras Clave: Uso de redes sociales, desempeño académico, estudiantes

\section{SUMMARY}

Participation in social networks is increasingly common among young people in our community. It is considered that this use can affect the academic performance precisely in those who perform academic tasks and particularly in university students. Objective: To determine if the use of social networks influences the academic performance of the students of the III Cycle of the Faculty of Human Medicine of the University of San Luis Gonzaga National University of Ica, 2017-I. Material and Methods: Seventy students participated, who voluntarily accepted the completion of a questionnaire that gathered information on three aspects: Use of social networks, sociodemographic aspects and addiction to social networks. In addition, there was a database of qualifications obtained by participants in an academic cycle, and the study variables were statistically correlated. Results: All participants said they participate in social networks (100\%), of the networks consulted, the so-called Facebook is the most used (70\%). An inverse relationship was found between the excessive use of social networks and the academic performance of the participants $(p=0.000)$. Conclusions: There is an inversely proportional relationship between the use of social networks and academic performance in students of the third cycle of the Faculty of Human Medicine of the National University San Luis Gonzaga de Ica. 2017-I, the greater the use of social networks, the lower the academic performance.

Keywords: Use of social networks, academic performance, students. 


\section{INTRODUCCIÓN}

La irrupción de las tecnologías digitales ha contribuido a la formación de importantes redes sociales en internet. Las plataformas tecnológicas más importantes son, por su volumen y cantidad de participantes, Facebook, Twitter, Myspace y Linked-in, y cada una de estas tecnologías de red social apunta a diferentes públicos y formas de uso. Dichas plataformas tecnológicas facilitan la aparición de las redes sociales online, creándose así verdaderas comunidades virtuales que permiten a diversos individuos y grupos relacionarse e interactuar socialmente dentro de un sistema común. Investigadores e instituciones han venido mostrando en los últimos años una creciente preocupación por el uso de Internet entre los jóvenes y adolescentes. Su impacto a nivel psicológico y conductual se traduce a menudo en la aparición de conductas de riesgo, fracaso escolar y problemas familiares (San Martín; 2010) (1).

Actualmente el ingreso de las nuevas tecnologías de información y comunicación (TIC) a la vida cotidiana tales como internet, vídeo juegos, móviles entre otros, han transformado la vida de los hombres, contribuyendo al desarrollo social individual y colectivo, pero afectando a la misma vez el proceso de sociabilización cara a cara (Oliva ,et al; 2012) (2).

En el mundo, las redes más utilizadas según el número de millones de usuarios son Facebook (1,500 millones), Wathsapp (900 millones), Messenger (800 millones) e Instagram (400 millones). En el Perú, la cifra oficial de peruanos en Facebook supera los 11 millones de usuarios únicos. Los números parecen más que atractivos. Asimismo, se señala que $65 \%$ de los internautas del país utiliza regularmente entre 3 y 6 redes sociales, y su participación activa y frecuente en el mundo virtual no es una moda pasajera sino un hecho concreto que propicia un mejor acercamiento entre las organizaciones y las audiencias. Si miramos los datos diarios de ingreso a la red social, encontraremos que 7,8 millones de usuarios residentes en el Perú acceden a la plataforma Facebook. De ese total, 5,2 millones se conectan a través de dispositivos móviles (El Comercio; 2016) (3).

La influencia de las redes sociales en los estudiantes es un problema social que día a día va incrementando. Se afirma que existe un alto índice de estudiantes que presentan un bajo rendimiento escolar, inasistencia a clases, debido al dominio que las redes sociales están teniendo en sus estudios, por lo que es necesario analizar cuáles fueron los factores que desencadenaron esta problemática. Ésta se ha convertido en un gran problema que está afectando a su desarrollo estudiantil. Entre los factores que encontramos está el pasar demasiado tiempo inmerso en las redes sea dentro o fuera de las clases (Mejía V; 2015) (4).

En este contexto, surge la necesidad de analizar en qué medida el uso de las redes sociales influye en el rendimiento académico de los estudiantes, considerando que hoy en la actualidad las redes sociales cumplen un papel importante en la vida de los universitarios, quienes pasan horas de horas en el celular o el computador interactuando con herramientas cibernéticas como son el Facebook, Twitter, Instagram, entre otros. Si bien es cierto que el internet ha revolucionado a la educación mediante la tecnología de la información y comunicación, existen factores negativas en el uso del internet, como es el caso de la adicción a las redes sociales, lo cual puede dar como resultado un bajo rendimiento en estudiantes quienes se dedican sólo a "chatear" en las redes sociales, descuidando sus estudios profesionales.

En el estudio se determinó el uso excesivo de redes sociales en alumnos universitarios del pre grado de la Facultad de Medicina 
Humana de la Universidad Nacional San Luis Gonzaga de Ica y se correlacionó dicha información con los promedios de calificación alcanzada por los participantes en un ciclo académico.

\section{MATERIALY MÉTODOS}

Tipo: El estudio realizado es de tipo prospectivo, transversal, observacional, descriptivo

Población y Muestra: La población la constituyeron todos los estudiantes del III Ciclo de la Facultad de Medicina de la Universidad San Luis Gonzaga de Ica, Perú que culminaron el periodo académico 2017-I, haciendo una población de 72 estudiantes.

Como muestra se tomó el íntegro de los estudiantes, agrupando a toda la población para la obtención de mayores resultados en el estudio, excluyendo a 2 estudiantes quienes estuvieron ausentes al momento de aplicar el instrumento de recolección de datos.

\begin{tabular}{|c|c|c|c|c|}
\hline \multirow{2}{*}{$\begin{array}{l}\text { Uso excesivo de las redes } \\
\text { sociales }\end{array}$} & \multicolumn{3}{|c|}{ Bondad y ajuste de chi cuadrado } & \multirow[b]{2}{*}{$\begin{array}{c}\text { Porcentaje } \\
(\%)\end{array}$} \\
\hline & Observado & Esperado & Residual & \\
\hline \multirow{4}{*}{$\begin{array}{l}\text { Adicción ( } 0-40) \\
\text { Abuso }(41-56) \\
\text { uso normal }(57-90) \\
\text { Total }\end{array}$} & 5 & 23,3 & $-18,3$ & 7,1 \\
\hline & 16 & 23,3 & $-7,3$ & 22,9 \\
\hline & 49 & 23,3 & 25,7 & 70,0 \\
\hline & 70 & & & 100,0 \\
\hline
\end{tabular}

El promedio ponderado final del rendimiento académico prevalente fue regular (66/70)
Quedando conformada la muestra por 70 estudiantes.

Técnicas de recolección de datos: Para la recolección de datos se utilizaron las técnicas de la entrevista y la encuesta.

Instrumento de recolección de datos: Se utilizó 1 instrumento recolección de información, con datos sociodemográficos y el cuestionario ARS de adicción a las redes sociales elaborado por Escurra M. y Salas E. (2014) (5), el cual ha sido validado por juicios de expertos, con una confiabilidad Alfa de Cronbach de 0,95.

\section{RESULTADOS.}

A la aplicación del cuestionario uso de las redes sociales se encontró que fue prevalente el uso normal (49/70) $70,0 \%$ seguido de abuso (16/70) $22,9 \%$ y en menor prevalencia adicción (5/70) 7,1\%.
$94,3 \%$ y en menor prevalencia fue malo y bueno (2/70) $2,9 \%$ respectivamente

\begin{tabular}{lcccc}
\hline \multicolumn{1}{c}{ Rendimiento académico } & \multicolumn{3}{c}{ Bondad y ajuste de chi cuadrado } & Porcentaje \\
\cline { 2 - 4 } & Observado & Esperado & Residual & $(\%)$ \\
\hline Malo $(0-10)$ & 2 & 23,3 & $-21,3$ & 2,9 \\
Regular $(10-15)$ & 66 & 23,3 & 42,7 & 94,3 \\
Bueno $(15-18)$ & 2 & 23,3 & $-21,3$ & 2,9 \\
Excelente $(18-20)$ & 0 & 23,3 & $-23,3$ & 0,0 \\
Total & 70 & & & 100,0 \\
\hline
\end{tabular}

Se encontró que la puntuación para el uso excesivo de las redes sociales estuvo comprendida entre 31 y 92 puntos con un promedio de 66,1 \pm 15,2 (uso normal) mientras que el rendimiento académico alcanzó puntuaciones de 7,425 y 15,083 puntos con un promedio de 12,5 $\pm 1,3$ (regular); por lo que con un p-valor $=0,020$ podemos concluir que existe relación significativa entre el uso excesivo de las redes sociales y el rendimiento académico de los estudiantes del III ciclo de la Facultad de Medicina Humana de la Universidad Nacional San Luis Gonzaga de Ica. A la determinación 
de la direccionalidad de las variables se encontró una correlación negativa débil $(r=-$ 0,278); es decir que a mayor uso de las redes sociales menor será el rendimiento académico o la inversa a menor uso de las redes sociales mayor será el rendimiento académico.

\begin{tabular}{|c|c|c|c|c|c|c|c|c|c|c|c|}
\hline \multicolumn{8}{|c|}{ Estadística descriptiva } & \multirow{2}{*}{\multicolumn{4}{|c|}{$\begin{array}{l}\text { Correlación de } \\
\text { Spearman }\end{array}$}} \\
\hline Adic & ión a & s redes & ciales & & $\operatorname{dimi}$ & to acad & ico & & & & \\
\hline $\begin{array}{c}\text { Media } \\
66,1\end{array}$ & $\begin{array}{l}\text { D.S } \\
15,2\end{array}$ & $\begin{array}{l}\text { Mínimo } \\
31\end{array}$ & $\begin{array}{l}\text { Máximo } \\
92\end{array}$ & $\begin{array}{c}\text { Media } \\
12,5\end{array}$ & $\begin{array}{l}\text { D.S } \\
1,3\end{array}$ & $\begin{array}{l}\text { Mínimo } \\
7,425\end{array}$ & $\begin{array}{l}\text { Máximo } \\
15,083\end{array}$ & $\begin{array}{c}\mathrm{T} \\
-2,143\end{array}$ & $\begin{array}{l}g l \\
69\end{array}$ & $\begin{array}{c}r \\
-0,278\end{array}$ & $\begin{array}{c}p \text {-valor } \\
0,020\end{array}$ \\
\hline
\end{tabular}

Fuente: Visor de resultados IBM SPSS Statistics versión 22

\section{DISCUSIÓN}

Muchos de los conocimientos en neurociencia y técnicas para afianzar el proceso de enseñanza aprendizaje han concitado la atención de eminentes filósofos y pedagogos como Mario Bunge a finales del siglo XX, hoy sin embargo debemos asumir que dentro del proceso está presente un factor distractivo que puede ser altamente perjudicial. El uso que puede terminar en dependencia, de redes sociales a través de vehículos digitales que son cada día más asequibles, sin demostrarse su real necesidad. La creciente industria de estos dispositivos no conoce de límites y solo busca posicionarse en el mercado como un producto de primera necesidad. Nunca como antes, se ha creado en los consumidores la sensación ficticia sobre la necesidad de "estar comunicado", consideración que puede seguramente debatirse extensamente. En el presente estudio se ha tenido como propósito determinar la relación entre el uso excesivo de las redes sociales y el rendimiento académico de los estudiantes del III ciclo de la Facultad de Medicina Humana de la Universidad San Luis Gonzaga de Ica. Diversa bibliografía contemporánea refiere haber realizado sus estudios con muestras mayores al nuestro. En nuestro estudio, se encontró que el $100,0 \%$ de los encuestados manifestaron utilizar redes sociales, en contraste no todos los estudios revisados dan cuenta de un uso total en toda la muestra como en nuestro caso. No obstante nuestros resultados coinciden con los de Jeffrey Mingle et al (6), quienes también coincidieron en que las redes más empleadas son Facebook y WhatsApp. El medio prevalente por el que se conecta a las redes sociales fue cabinas de internet $100,0 \%$; seguido de a través del celular $87,1 \%$; en mi casa $(57 / 70) 81,4 \%$ y un solo caso en computadoras de la universidad $1,4 \%$. Al respecto, estudios similares en el país de Ghana no se hace referencia a una posesión tan común de dispositivos móviles en jóvenes estudiantes, no obstante son contemporáneos a nuestra muestra. En el análisis del número de horas que accede por semana a las redes sociales, se reportaron algunos estudiantes con 35 horas $(n=4) ; 56$ horas $(n=1) ; 70$ horas $(n=2)$; y 82 horas $(n=1)$. Estos resultados coinciden con los obtenidos por Tamez P ( 7),quien evidencio uso de redes sociales en estudiantes desde las 10 horas hasta las 100 horas semanales, dichos casos pueden alcanzar un uso de 14 horas diarias, Orellana $L$ (8),por su parte promedia un uso de dos horas diarias, deduciéndose en este punto que aún en nuestro país, es amplio el intervalo de horas que puede dedicarse al uso de redes sociales, para nuestro estudio el número de horas que acceden a las redes sociales estuvo comprendido entre una hora y 82 horas con una mediana de 18 horas $\mathrm{IC}_{95 \%}=[14-21]$.

Con un p>0,05 no se encontró variables socio demográficas asociados al uso excesivo de las redes sociales sin embargo a continuación se detallan algunas diferencias numéricas de adicción y abuso pero que no alcanzaron una diferencia estadística significativa, estos 
resultados coinciden con la literatura actual, en la que no se ha podido relacionar con significancia estadística, parámetros socio demográficos con el uso o dependencia de redes sociales, no obstante la cantidad de muestra mayor a nuestro estudio.

Según la edad fue prevalente la condición de abuso de las redes sociales en el grupo de 17 a 23 años (15/64) 23,4\% y adicción (5/64) $7,8 \%$; mientras que en el grupo de 24 a 30 años solo se presentó un caso de abuso (1/6) $16,7 \%(p=0,556)$, Al respecto, Orellana $L$ (8), alcanza a concluir que a mayor edad en la muestra, el uso de redes sociales se hace moderado. No se describe en la literatura consultada la condición de abuso de las redes sociales asociada a algún género en particular, aunque el estudio de Jonna M. Leyrer-Jackson (9), afirma que el mayor uso de redes sociales se realiza por parte del género femenino, en nuestro estudio por el contrario es el género masculino el que alcanzo valores mayores en cuanto a abuso (6/24) 25,0 \% y adicción (2/24) 8,3\% $(p=0,904)$ en el uso de redes sociales. No se reporta en la bibliografía las condiciones de Estado civil, procedencia geográfica, que pudiera compararse con nuestros resultados. Para dichos parámetros nuestro estudio resolvió que mayoritariamente los solteros reportan mayores cifras de abuso de las redes sociales, 23,9\% y adicción 7,5\%; comparados a los casados ( $p=0,333)$. Respecto a la procedencia, la condición urbana reporta también porcentajes superiores comparada a la rural $23,7 \%$ y adicción (5/59) 8,5\% frente a solo abuso de las redes sociales (2/11) $18,2 \%, \sin$ reportarse adicción ( $p=0,351)$.

Respecto al último parámetro consignado en nuestro estudio, el estado laboral, fue prevalente la condición de abuso de las redes sociales en los estudiantes que no trabajan (15/62) 24,2\% y adicción (4/62) 6,5\%; mientras que en el grupo que trabaja se encontró abuso y adicción a las redes sociales $12,5 \%$ respectivamente $(p=0,659)$. Finalmente, este estudio encontró que la puntuación para el uso excesivo de las redes sociales estuvo comprendida entre 31 y 92 puntos con un promedio de $66,1 \pm 15,2$ (uso normal) mientras que el rendimiento académico alcanzó puntuaciones de 7,425 y 15,083 puntos con un promedio de 12,5 $\pm 1,3$ (regular); por lo que con un $p$-valor $=0,020$ podemos concluir que existe relación significativa entre el uso excesivo de las redes sociales y el rendimiento académico de los estudiantes del III ciclo de la Facultad de Medicina Humana de la Universidad Nacional San Luis Gonzaga de Ica. A la determinación de la direccionalidad de las variables se encontró una correlación negativa débil $(r=$ 0,278 ); es decir que a mayor uso de las redes sociales menor será el rendimiento académico o la inversa a menor uso de las redes sociales mayor será el rendimiento académico. Nuestros resultados por tanto, coinciden con lo hallado en la literatura, Hernández G. et al (10), concluye igualmente que el uso de redes sociales genera un bajo rendimiento académico, Araujo $E$ (11), afirma en su estudio que un $90 \%$ de estudiantes solo alcanza calificaciones iguales o menores a 20/40; en ese mismo sentido Owusu Acheaw et al (12), concluyeron que el uso de redes sociales afecto el trabajo académico de los estudiantes negativamente. No obstante estos resultados contrastan por lo obtenido con Pavón M.(13), quien determino que no existió en su estudio relación estadísticamente significativa entre el tiempo que los estudiantes dedican a las redes sociales y su rendimiento académico por lo que se concluyó que las redes sociales no inciden en el rendimiento académico de los alumnos.

\section{CONCLUSIONES}

1. Existe relación inversamente proporcional entre el uso excesivo de las redes sociales y el rendimiento académico de los estudiantes del III ciclo de la Facultad de Medicina Humana de la Universidad 
Nacional San Luis Gonzaga de Ica. (pvalor $=0,020)$. A mayor uso de redes sociales, el rendimiento académico es menor.

2. La utilización de las redes sociales en los estudiantes del III ciclo de la Facultad de Medicina Humana de la Universidad Nacional San Luis Gonzaga de Ica, es de uso normal con $70 \%$, abuso $22,9 \%$, y adicción a las redes sociales con $7,1 \%$.

3. El rendimiento académico de los estudiantes del III ciclo de la Facultad de Medicina Humana de la Universidad Nacional San Luis Gonzaga de Ica, es regular con $94,3 \%$, buen rendimiento $2,9 \%$, y mal rendimiento académico con $2,9 \%$.

\section{RECOMENDACIONES}

1. Se recomienda realizar estudios similares con muestras mayores y que comprendan tanto otros ciclos académicos como otras escuelas profesionales.

2. Se recomienda también realizar estudios similares en otros niveles de estudios superiores como el Posgrado.

3. Se sugiere un trabajo conjunto en la Universidad en general para analizar y evaluar la viabilidad de programas que resalten los beneficios de las redes sociales, las aprovechen mejor y ayuden a los usuarios a un adecuado empleo de las mismas.

4. Considero que de momento, la utilidad de redes sociales debe estar claramente restringida a los horarios fuera de clase.

\section{Correspondencia:}

Mg. Juana Maribel Arroyo Hernández

Correo Electrónico:

marybelyta@hotmail.com

\section{REFERENCIAS BIBLIOGRÁFICAS.}

1. San Martín L, Mujica S. Redes sociales: La nueva forma de comunicación de los jóvenes universitarios. Eighth LACCEI Latin American and Caribbean Conference for Engineering and Technology (LACCEI2010): "Innovation and Development for the Americas", June 1-4, 2010, Arequipa, Peru.

2. Oliva $A$, Hidalgo $M$, Moreno $C$, Jiménez L, Jiménez A, Antolín L, et al. Uso y riesgo de adicciones a las nuevas tecnologías entre los adolescentes y jóvenes Andaluces. Trabajo de Investigación. Andalucía, España. Departamento de Psicología y de Educación, Universidad de Sevilla; 2012.

3. Diario El Comercio. Redes sociales para empresas: Tendencias 2016. Lima, Perú;2016

4. Mejía V. Análisis de la influencia de las redes sociales en la formación de los jóvenes de los colegios del Cantón Yaguachi. Tesis pregrado. Guayaquil, Ecuador. Universidad de Guayaquil. Ecuador; 2015.

5. Escurra M, Salas E. Construcción y validación del cuestionario de adicción a las redes sociales. Lima, Perú .Rev.Liberabit.2014; 20(1): 73-91.

6. Mingle J, Adams M. Social Media Network Participation and academic performance in Senior High Schools in Ghana. University of Nebraska - Lincoln. 2015; 7(21).

7. Tamez P. Adicción a la red social de Facebook y su incidencia en el rendimiento académico de estudiantes de la Preparatoria 20 de la Universidad Autónoma de Nuevo León. Tesis Maestría. Monterrey, México. Facultad de Psicología, Universidad Autónoma de Nuevo León; 2012. 
8. Orellana L. Uso de internet por jóvenes universitarios de la Facultad de Ciencias Sociales de la Universidad Nacional Federico Villarreal. Tesis Maestría. Lima, Perú. Facultad de Letras y Ciencias Humanas, Universidad Nacional Mayor de San Marcos; 2012.

9. Jonna M, Leyrer J, Ashley W. The associations between social-media use and academic performance among undergraduate students in biology. Journal of Biological Education;2017

10. Hernández G, Castro A. Influencia de las redes sociales de internet en el rendimiento académico del área de informática en los estudiantes de los grados $8^{\circ}$ y $9^{\circ}$ del instituto promoción social del norte de Bucaramanga. Tesis Maestría. Ibagué, Colombia. Universidad del Tolima; 2014.
11. Araujo E. Indicadores de adicción a las redes sociales en universitarios de Lima, Perú. Rev. RIDU .2016; 10(2).

12. Owusu A. et al. Use of social Media and its impact on academic performance of Tertiary Institution students: A Study of Students of Koforidua Polytechnic, Ghana. Journal of Education and Practice.2015; $6(6)$.

13. Pavón M. EL uso de las redes sociales y sus efectos en el rendimiento académico de los alumnos del Instituto San José, El Progreso, Yoro-Honduras. Tesis Maestría. Guatemala. Facultad de Humanidades .Universidad Rafael Landívar; 2015.

Recibido: 03/01/18

Aprobado para Publicación: 05/04/18 